\title{
Tuberculosis in infants less than 3 months of age
}

\author{
H S Schaaf, R P Gie, N Beyers, N Smuts, P R Donald
}

\begin{abstract}
The clinical and radiological features in $\mathbf{3 8}$ infants less than 3 months of age with tuberculosis proved by culture are described and may aid early diagnosis of this often fatal condition. Respiratory symptoms, cough in $33(87 \%)$ and tachypnoea in $31(82 \%)$, were the commonest presenting symptoms. Twenty five infants $(66 \%)$ had hepatomegaly and $20(53 \%)$ splenomegaly. Mantoux testing gave an induration of $>15 \mathrm{~mm}$ in three of $17(18 \%)$ infants. In a further five a Tine test gave confluent response. Chest radiography in 27 infants showed miliary tuberculosis in seven $(26 \%)$ and hilar or paratracheal adenopathy in $14(52 \%)$ and $10(37 \%)$ respectively. Compression of either the bronchi or trachea or both was noted in 15 $(56 \%)$. Detection of this complication was aided by high kilovolt radiographs. A culture of Mycobacterium tuberculosis was obtained from gastric aspirate in $\mathbf{3 5}$ $(92 \%)$ infants, but positive cultures were also obtained from cerebrospinal fluid, tracheal or bronchial aspirate and liver and lymph node biopsy. Of 30 mothers evaluated seven (23) had previously unsuspected pulmonary tuberculosis.

(Arch Dis Child 1993; 69: 371-374)
\end{abstract}

The morbidity and mortality of tuberculosis in childhood is at its highest in infancy and the frequent occurrence of disseminated forms of tuberculosis such as miliary tuberculosis and tuberculous meningitis is partly responsible for this poor prognosis. ${ }^{1}$ Furthermore many case reports emphasise the sometimes atypical presentation of tuberculosis in infancy, so that whether of congenital or postnatal origin, diagnostic delay contributes to the poor outcome. ${ }^{2-5}$

In this study we describe the clinical and radiological features of tuberculosis proved by culture presenting in 38 children at an age of less than 3 months and stress those features that may aid early diagnosis and treatment.

\section{Patients and methods}

This study was carried out in the paediatric wards and outpatient department of Tygerberg Hospital, a tertiary care hospital situated in the south western Cape Province of South Africa, an area with a particularly high incidence of tuberculosis $\left(>600 / 100000\right.$ in 1990). ${ }^{6}$ Nine infants were identified during a prospective evaluation of childhood tuberculosis undertaken during 1991, and a further 29 infants less than 3 months of age with tuberculosis proved by culture seen between 1987 and 1990 were identified from a register of cases proved by culture.

A history of contact with adult pulmonary tuberculosis, the presenting symptoms and their duration, and clinical features such as lymphadenopathy, respiratory signs, and the presence of hepatosplenomegaly were noted. Tuberculin testing was either by Mantoux test 5 units purified protein derivative or Tine test (Lederle) with an induration of $>15 \mathrm{~mm}$ or a confluent reaction respectively being regarded as significant.

The chest radiographs of $27(71 \%)$ of the 38 infants were assessed systematically by a panel consisting of all the authors. Particular attention was paid to the presence of miliary tuberculosis, the presence of hilar or paratracheal adenopathy, narrowing of large airways, and signs of air trapping. In a further nine children a radiologist's report was available. In eight infants, in whom narrowing of the large airways was clinically suspected, high kilovolt chest radiographs were taken. Six of the infants who presented during the neonatal period have been briefly reported previously. ${ }^{78}$

The study was approved by the ethical committee of the Faculty of Medicine of the University of Stellenbosch.

\section{Results}

The median age at presentation was 65 days with the youngest child being 8 days. The male to female ratio was $24: 14$. The median duration of symptoms before the diagnosis of tuberculosis was considered and antituberculous treatment started was 14 days (range 1-60 days). The duration of symptoms were less than one week in $13(34 \%)$ of the infants.

Certain presenting features are summarised in table 1 . The weight of $15(42 \%)$ of the infants was below the third centile. Tachypnoea (respiratory rate $>50 / \mathrm{min}$ ) was present in $31(82 \%)$ of the infants. Tuberculous meningitis was clinically suspected in four infants and was confirmed by special investigations. Mycobacterium tuberculosis was cultured from the cerebrospinal fluid in two, one had miliary tuberculosis involving the meninges demonstrated on postmortem examination, and in one infant with disseminated tuberculosis, computed tomography showed hydrocephalus and a basal exudate typical of tuberculous meningitis. ${ }^{9}$

A tuberculin test was done and read in 31 infants and was significant in eight $(26 \%)$. A Mantoux test resulted in an induration $>15 \mathrm{~mm}$ in three of 17 infants and a Tine test gave a confluent reaction in a further five infants. 
The findings of the 27 chest radiographs available for evaluation by the authors are set out in table 2. A miliary picture was present in seven $(26 \%)$ and signs of large airway compression in $11(41 \%)$. In eight infants high kilovolt chest radiographs were also taken and revealed airway compression in a further four infants. Thus of the 27 infants, 15 $(56 \%)$ had compression of the large airways (figs 1 and 2). All nine chest radiographs, for which only a radiologist's report was available, showed some form of opacification either

Table 1 Presenting features in 38 infants less than 3 months of age with culture proved tuberculosis; values are number (\%)

$\begin{array}{lc}\text { History } & \\ \text { Household contact with adult pulmonary } & \\ \text { tuberculosis } & 19(50) \\ \text { Cough } & 33(87) \\ \text { Wheezing } & 15(40) \\ \text { Respiratory signs } & 31(82) \\ \text { Tachypnoea } & 6(16) \\ \text { Stridor } & 13(34) \\ \text { Wheeze } & 17(45) \\ \text { Crepitations } & 2(5) \\ \text { Bronchial breathing } & 15(42) \\ \text { Other signs } & 25(66) \\ \text { Weight <3rd centile for aget } & 20(53) \\ \text { Hepatomegaly } & 3(8) \\ \text { Splenomegaly } & 4(11) \\ \text { Prolonged jaundice } & 3(8) \\ \text { Tuberculous meningitis } & \end{array}$

$\star$ Respiratory rate $>50$ per minute.

tWeight available in 36 infants.

Table 2 Findings on routine chest radiography of 27 infants less than 3 months of age with proved tuberculosis * values are number (\%)

Miliary tuberculosis

Lymphadenopathy

Hilar

Paratracheal

Large airway compression

Bronchial

Tracheal

Airtrapping

Lobar consolidation

Bronchopneumonia

Ghon's focus

Lobar collapse

Pleural effusion

Normal radiograph

$14(52)$

10 (37)

$11(41)$

$2(7)$
$15(56)$

$15(56)$
$14(52)$

$5(19)$

2 (7)

$4(15)$

2 (7)

1 (4)

^ Most infants had more than one abnormal finding on chest radiography.

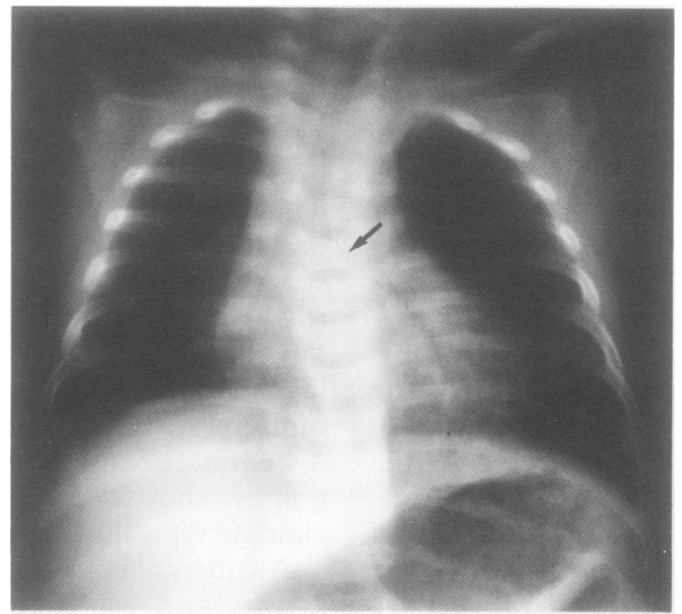

Figure 1 Chest radiograph of a 2.5 month old infant with bronchopneumonia, airtrapping, narrowing of large airways (arrow), and right hilar lymphadenopathy.

Mantoux test was $8 \mathrm{~mm}$ indurated and gastric aspirates were culture positive for $\mathrm{M}$ tuberculosis.

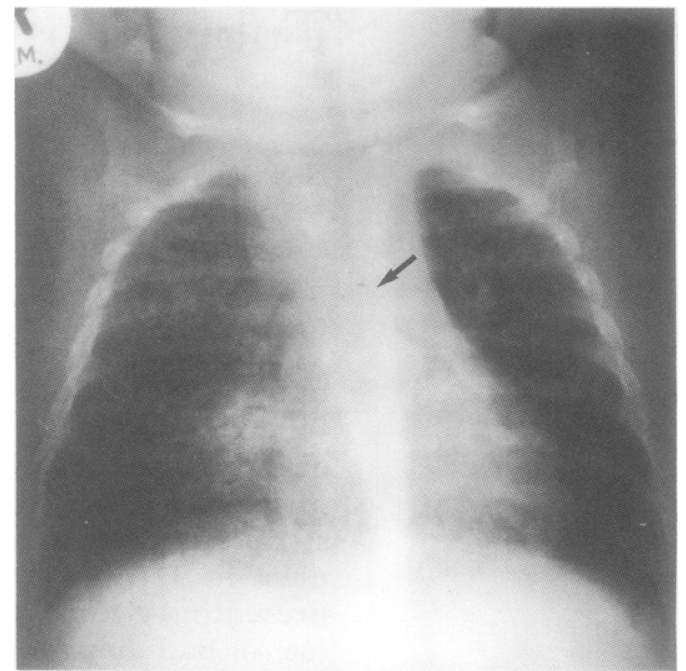

Figure 2 High kilovolt chest radiograph of a 2 month old infant shows narrowing of large airways (arrow) in the absence of obvious lymphadenopathy. Mantoux test was $17 \mathrm{~mm}$ indurated and a gastric aspirate culture positive for $\mathrm{M}$ tuberculosis.

bronchopneumonic or lobar. Three were reported to show miliary tuberculosis, four lymphadenopathy (two paratracheal and two parahilar), and one bronchial compression.

Sources of positive cultures for $M$ tuberculosis are listed in table 3. $M$ tuberculosis was cultured from the gastric aspirate of 35 of the 38 babies. Other sources of positive cultures included cerebrospinal fluid, liver biopsy, and tracheal and bronchial aspirates.

Because of suspicion of tuberculosis in the infants, 30 of the mothers were evaluated for active tuberculosis. In seven mothers (23\%) previously unsuspected pulmonary tuberculosis was diagnosed on chest radiography and confirmed by sputum culture of $M$ tuberculosis. A further four also had active pulmonary tuberculosis confirmed by sputum culture of $M$ tuberculosis but the diagnosis had been made previously. In addition one mother whose chest radiograph was clear was only subsequently found to have urogenital tuberculosis.

It is difficult and perhaps no longer of practical importance to apply Beitzke's criteria for congenital tuberculosis as the majority of infants survived and were not separated from their mothers at birth. ${ }^{3}$ However, taking into consideration maternal urogenital tuberculosis without pulmonary involvement, an absence of other close contacts for

Table 3 Origin of cultures positive for $\mathrm{M}$ tuberculosis in infants less than 3 months of age

\begin{tabular}{lc}
$\begin{array}{l}\text { Origin of culture positive } \\
\text { for M tuberculosis }\end{array}$ & $\begin{array}{c}\text { No of } \\
\text { infants }\end{array}$ \\
\hline GA only & 28 \\
GA+CSF & 2 \\
GA+tracheal or bronchial aspirate & 2 \\
GA+biopsy (lymph node/bone marrow) & 2 \\
GA+liver biopsy + bronchial aspirate & 1 \\
Liver biopsy & 1 \\
CSF+ bronchial aspirate & 1 \\
Tracheal aspirate & 1 \\
Total infants & 38 \\
Total positive cultures & 47
\end{tabular}

$\mathrm{CSF}=$ cerebrospinal fluid, $\mathrm{GA}=$ gastric aspirate. 
pulmonary tuberculosis, an age at presentation of less than 4 weeks and prolonged neonatal jaundice as a presenting symptom, only seven infants can confidently be said to have had congenital tuberculosis.

HIV infection has only recently been detected in the western Cape Province and in a 1991 survey the prevalence rate in women attending antenatal clinics was $0.37 \%(95 \%$ confidence interval $0 \cdot 18$ to $0 \cdot 57) .{ }^{10}$ All enzyme linked immunosorbent assay (ELISA) tests for HIV done during the prospective evaluation of infants were negative.

Five $(13 \%)$ of the infants died. Of these deaths three were probably due to the disseminated tuberculosis, one developed pneumococcal meningitis in addition to tuberculous meningitis, and one developed a Klebsiella pneumoniae septicaemia.

\section{Discussion}

A prerequisite for the early diagnosis of tuberculosis in childhood is a high index of suspicion. ${ }^{11}$ At no age is this more important than during infancy when the consequences of a missed or delayed diagnosis are likely to be considerably more serious than at a later age. Despite the fact that our hospital lies within an area with a particularly high incidence of tuberculosis a median period of 14 days elapsed between the recognition of the first symptoms, the diagnosis of tuberculosis, and initiation of treatment.

The non-specific nature of the symptoms and associated clinical signs of tuberculosis in early infancy have been repeatedly commented upon in the literature dealing with congenital tuberculosis. ${ }^{3-5}$ Our experience suggests none the less that a systematic evaluation of infants particularly when presenting with respiratory symptoms and signs or failure to thrive should lead to the consideration of tuberculosis.

First and foremost an evaluation of close contacts for possible pulmonary tuberculosis or other forms of tuberculosis is essential. ${ }^{3}$ Our experience with these young infants and older children $^{12}$ confirms the value of chest radiography with respect to the mother, but other family members and even hospital personnel should not be neglected.

Tachypnoea, crepitations, and bronchial breathing are common to a wide variety of neonatal and congenital infections. Wheeze and airway narrowing, however, have been repeatedly documented to occur in childhood tuberculosis, ${ }^{13} 14$ and the younger the child the more likely it is that these symptoms will be encountered.

Hepatosplenomegaly was a common finding in this and earlier studies of congenital tuberculosis ${ }^{3}$ and its detection should lead to consideration of possible disseminated tuberculosis. It should also be borne in mind that prolonged jaundice may be caused by tuberculosis, a fact well known to earlier workers but neglected in recent textbooks. We have recently reported on this finding. ${ }^{8}$

Tuberculous meningitis is usually considered a disease of children older than 6 months, but our experience illustrates that should infection take place at a very young age that tuberculous meningitis may be seen in infants less than 3 months of age not only as a serous reaction complicating miliary tuberculosis but in its classical form with hydrocephalus accompanied by a basal exudate.

In contrast to other reports of congenital or neonatally acquired tuberculosis, extrapulmonary lymphadenopathy was relatively uncommon in this study and we also did not encounter tuberculous skin lesions, biopsy of which may aid the diagnosis of tuberculosis. ${ }^{15}$

With a single exception all of the chest radiographs evaluated or for which a radiologist's report was available were abnormal. The single most common abnormality seen was airtrapping particularly in the region of the right lower and middle lobes, while hilar adenopathy and lobar opacification were present in more than half of the radiographs evaluated. A careful evaluation of radiographs for possible miliary tuberculosis requires no emphasis other than to repeat the warning that a normal chest radiograph does not exclude miliary tuberculosis and that miliary changes may develop within several days. ${ }^{16}$

Given the frequency of wheezing and stridor in these infants the finding of narrowing of the large airways in 15 of the 27 radiographs available for evaluation was not surprising. ${ }^{4}$ Even in children without obvious lymphadenopathy resort to high kilovolt radiographs aided the diagnosis of tuberculosis by the detection of the narrowing of large airways in four infants.

The commonest source of confirmatory cultures of $M$ tuberculosis in these infants was gastric aspirate but we support the contention that use should be made of all available body fluids and biopsies to confirm the diagnosis. ${ }^{17}$

Despite the frequency with which negative results are obtained tuberculin testing in early infancy, preferably using a Mantoux test, is an essential part of the evaluation of any child suspected of having tuberculosis. In the neonate our own experience ${ }^{7}$ and that of others ${ }^{3}$ is that a negative result is the rule. In older infants however tuberculin testing becomes more valuable. In our case the administration of BCG in approximately $85 \%$ of infants ${ }^{18}$ confounds the interpretation of a positive tuberculin test but even under these circumstances a strongly positive result giving an induration of $>15 \mathrm{~mm}$ should not be ignored. ${ }^{19}$

The occurrence of other infections in addition to tuberculosis is not uncommon and has been reported by others ${ }^{2021}$ and should not deter the clinician from starting or continuing antituberculous treatment in suspicious circumstances. A possible source of confusion when treating for other bacterial infections is that the practice of using an aminoglycoside for possible Gram negative infections may lead to a temporary improvement in the condition of an infant with tuberculosis and so delay the diagnosis of tuberculosis.

The majority of our patients were treated for six months with isoniazid $10 \mathrm{mg} / \mathrm{kg} /$ day, 
rifampicin $10 \mathrm{mg} / \mathrm{kg} / \mathrm{day}$, and pyrazinamide $25-30 \mathrm{mg} / \mathrm{kg} /$ day. We have not encountered any problems with toxicity despite the fact that many of the infants were malnourished and acutely ill at the time of diagnosis. All infants received pyridoxine $0.5 \mathrm{mg} /$ day as part of daily multivitamin supplement for the full duration of treatment.

This study of a large group of young infants with tuberculosis shows that with a high index of suspicion and the correct interpretation of chest radiographs, the disease can be diagnosed early in infants and lead to the early initiation of treatment and a lower mortality.

Prevention of tuberculosis in early infancy rests upon the early detection and treatment of tuberculosis in the expectant mother and other members of the infant's household. Persistent cough, night sweats, and recent loss of weight should lead to chest radiography, sputum culture and microscopy, and tuberculin testing, which has been shown to remain reliable during pregnancy, 22 although its value in areas of high incidence might be questioned.

This study was supported by the Doris and Harry Crossley fund and the South African Medical Research Council. We thank the medical superintendent of Tygerberg Hospital for permission to publish.

1 Rich AR. The influence of sex and age. The pathogenesis of tuberculosis. Springfield: Charles C Thomas, 1951: tuberculosis.

2 Snider DE, Bloch AB. Congenital tuberculosis. Tubercle 1984; 65: 81-2.

3 Hageman J, Shulman S, Schreiber M, Luck S, Yogev R Congenital tuberculosis: critical reappraisal of clinical findings and diagnostic procedures. Pediatrics 1980; 66: $980-4$
4 Myers JP, Perlstein PH, Light IJ, Towbin RB, Dinscoy HP, Dinscoy MY. Tuberculosis in pregnancy with fatal consenital infection. Pediatrics 1981; 67: 89-94.

5 Nemir RL, O'Hare D. Congenital tuberculosis. Review and diagnostic guidelines. Am 7 Dis Child 1985; 139: 284-7. diagnostic guidelines. Am fo Dis Child 1985; 139: 284-7. Steenekamp JHB. Tuberculosis control program

7 Schaaf HS, Smith J, Donald PR, Stockland B. Tuberculosis presenting in the neonatal period. Clin Pediatr (Phila) 1989; 28: 474-5.

8 Schaaf HS, Nel ED. Tuberculosis presenting as cholestatic jaundice in early infancy. $\mathcal{f}$ Pediatr Gastroenterol Nut 1992; 15: 437-9.

9 Bullock MR, Welchman JM. Diagnostic and prognostic features of tuberculosis meningitis on CT scanning. f Neurol Neurosurg Psychiatry 1982; 45: 1098-101.

10 Swanevelder R. Second national HIV survey of women attending antenatal clinics, South Africa, October/ November 1991. Epidemiological Comments 1992; 19: No-9.

11 Miller FJW. Intrathoracic tuberculosis and miliary spread. Tuberculosis in children. Edinburgh: Churchill Livingstone, 1982: 105-36.

12 Schaaf HS, Donald PR, Scott F. Maternal chest radiography as supporting evidence for the diagnosis of tuberculosis in childhood. $\mathcal{F}$ Trop Pediatr 1991; 37: 223-5.

13 Starke JR. Modern approach to the diagnosis and treatment of tuberculosis in children. Pediatr Clin North Am 1988; 35: 441-64.

14 Donald PR, Ball JB, Burger PR. Bacteriologically confirmed pulmonary tuberculosis in childhood. $S$ Afr Med 71985 ; pulmonary

15 McCray MK, Esterly MB. Cutaneous eruptions in congenital tuberculosis. Arch Dermatol 1981; 117: 460-4.

16 Palmer PES. Pulmonary tuberculosis - usual and unusual radiographic presentations. Semin Roentgenol 1979; 14: 204-43.

17 Snider DE, Rieder HL, Combs D, Bloch AB, Hayden $\mathrm{CH}$ Smith MHD. Tuberculosis in children. Pediatr Infect Dis 1988; 7: 271-8.

18 Unicef. Statistical tables. In: Grant JP, ed. The state of the world's children. New York: Oxford University Press, 1992: 69-96.

19 Niles RA. Puerperal tuberculosis with death of infant. $A m \mathcal{F}$ Obstet Gynecol 1982; 144: 131-2.

20 Brooke OG, Dow J, Hand TK. Escherichia coli meningitis and congenital tuberculosis in the same infant. Lancet 1977; i: 599.

21 Mucklow ES, Hallet RJ. Neonatal tuberculosis. Arch Dis Child 1986; 61: 1146.

22 Present PA, Comstock GW. Tuberculin sensitivity in pregnancy. Am Rev Respir Dis 1975; 112: 413-6. 